OPEN $\curvearrowright$ ACCESS

드 COMMUNICATIONS

ISSN 2056-9890

\section{Crystal structure of 3-bromo-9-ethyl-9H- carbazole}

\author{
Mykola Bezuglyi, ${ }^{a *}$ Gintare Grybauskaite, ${ }^{b}$ Gintautas \\ Bagdziunas $^{b}$ and Juozas Vidas Grazulevicius ${ }^{b}$

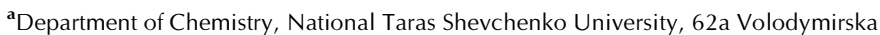 \\ st., Kyiv, Ukraine, and ${ }^{\mathbf{b}}$ Department of Polymer Chemistry and Technology, Kaunas \\ University of Technology, Radvilenu Road 19, LT-50254, Kaunas, Lithuania. \\ *Correspondence e-mail: nikolay_bezugliy@ukr.net
}

Received 29 July 2015; accepted 11 December 2015

Edited by G. S. Nichol, University of Edinburgh, Scotland

In the title compound, $\mathrm{C}_{14} \mathrm{H}_{12} \mathrm{BrN}$, the tricyclic ring system is essentially planar (r.m.s. deviation $0.026 \AA$ ). The carbon atoms of the ethyl group deviate from the mean plane by 0.148 (9) $\left(\mathrm{CH}_{2}\right)$ and $1.59(1) \AA\left(\mathrm{CH}_{3}\right)$. In the crystal, $\mathrm{H} \cdots \pi$ contacts [2.698-2.898 $\AA$ ] shorter than the van der Waals contact distance of $3.70 \AA$ are observed. A scalable to gram quantities selective synthesis of mono-bromine-substituted carbazole derivatives was developed.

Keywords: crystal structure; carbazole; $\mathrm{C}-\mathrm{H} \cdots \pi$ interactions.

CCDC reference: 1442215

\section{Related literature}

$\mathrm{N}$-substituted carbazole derivatives are important for anticancer research (Caulfield et al., 2002) and as materials for opto-electronic devices (Niu et al., 2011; Miyazaki et al., 2014; Grigalevicius et al., 2002). The crystal structure of 1,3,6,8tetrabromo-9-ethyl-9H-carbazole was reported by Bezuglyi et al. (2015).

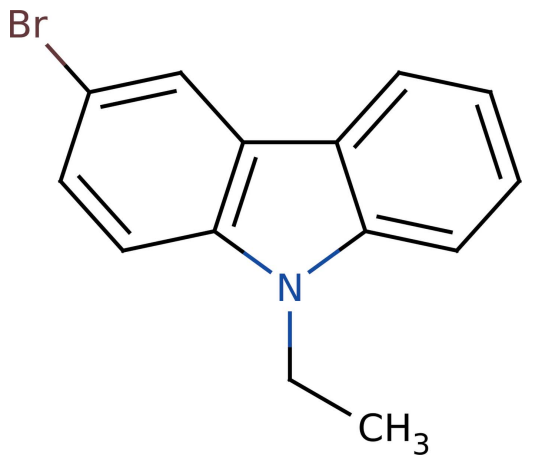

\section{Experimental}

2.1. Crystal data

$\mathrm{C}_{14} \mathrm{H}_{12} \mathrm{BrN}$

$M_{r}=274.16$

Orthorhombic, $\mathrm{Pbca}$

$a=15.263(16) \AA$

$b=7.745(8) \AA$

$c=20.41(2) \AA$

$V=2413(5) \AA^{3}$

$Z=8$

Mo $K \alpha$ radiation

$\mu=3.39 \mathrm{~mm}^{-1}$

$T=293 \mathrm{~K}$

$0.40 \times 0.09 \times 0.08 \mathrm{~mm}$

\subsection{Data collection}

Rigaku XtaLAB mini diffractometer

Absorption correction: multi-scan (REQAB; Rigaku, 1998)

$T_{\min }=0.450, T_{\max }=0.763$

8316 measured reflections 2721 independent reflections 1383 reflections with $F^{2}>2.0 \sigma\left(F^{2}\right)$ $R_{\text {int }}=0.056$

\subsection{Refinement}

$R\left[F^{2}>2 \sigma\left(F^{2}\right)\right]=0.078$

$w R\left(F^{2}\right)=0.236$

$S=1.05$

2721 reflections

145 parameters

$\mathrm{H}$-atom parameters constrained

$\Delta \rho_{\max }=1.37 \mathrm{e} \AA^{-3}$

$\Delta \rho_{\min }=-0.46 \mathrm{e} \AA^{-3}$

Table 1

Hydrogen-bond geometry $\left(\AA,{ }^{\circ}\right)$.

$C g 1$ are the centroids of the $\mathrm{N} 1 / \mathrm{C} 1 / \mathrm{C} 6 / \mathrm{C} 7 / \mathrm{C} 12$ and $\mathrm{C} 1-\mathrm{C} 6$ rings, respectively.

\begin{tabular}{lllll}
\hline$D-\mathrm{H} \cdots A$ & $D-\mathrm{H}$ & $\mathrm{H} \cdots A$ & $D \cdots A$ & $D-\mathrm{H} \cdots A$ \\
\hline $\mathrm{C} 8-\mathrm{H} 8 \cdots C g 1^{\mathrm{i}}$ & 0.93 & 2.81 & $3.637(7)$ & 149 \\
$\mathrm{C} 11-\mathrm{H} 11 \cdots C g 2^{\mathrm{ii}}$ & 0.93 & 3.01 & $3.922(8)$ & 167 \\
\hline
\end{tabular}

Symmetry code: (i) $-x+1, y+\frac{1}{2},-z+\frac{1}{2}$; (ii) $-x+\frac{3}{2}, y+\frac{1}{2}, z$.

Data collection: CrystalClear-SM Expert (Rigaku, 2011); cell refinement: CrystalClear-SM Expert; data reduction: CrystalClear-SM Expert; program(s) used to solve structure: SHELXS97 (Sheldrick, 2008); program(s) used to refine structure: SHELXL97 (Sheldrick, 2008); molecular graphics: ORTEP-3 for Windows (Farrugia, 2012); software used to prepare material for publication: CrystalStructure (Rigaku, 2010).

\section{Acknowledgements}

This research was supported by FP7 REGPOT-2012-2013-1 ICT project CEOSeR under grant agreement No 316010. The authors are grateful to Dr Vasyl Kinzhybalo from the Institute of Low Temperature and Structure Research, Polish Academy of Sciences, for valuable recommendations.

Supporting information for this paper is available from the IUCr electronic archives (Reference: NK2233).

\section{References}

Bezuglyi, M., Grybauskaite, G., Bagdziunas, G. \& Grazulevicius, J. V. (2015). Acta Cryst. E71, o373.

Caulfield, T., Cherrier, M. P., Combeau, C. \& Mailliet, P. (2002). Eur. Patent No. 1253141

Farrugia, L. J. (2012). J. Appl. Cryst. 45, 849-854. 


\section{data reports}

Grigalevicius, S., Ostrauskaite, J., Grazulevicius, J. V., Gaidelis, V., Jankauskas, V. \& Sidaravicius, J. (2002). Mater. Chem. Phys. 77, 281-284.

Miyazaki, T., Shibahara, M., Fujishige, J., Watanabe, M., Goto, K. \& Shinmyozu, T. (2014). J. Org. Chem. 79, 11440-11453.

Niu, F., Niu, H., Liu, Y., Lian, J. \& Zeng, P. (2011). RSC Adv. 1, 415-423.
Rigaku (1998). REQAB. Rigaku Corporation, Tokyo, Japan.

Rigaku (2010). CrystalStructure. Rigaku Corporation, Tokyo, Japan.

Rigaku (2011). CrystalClear-SM Expert. Rigaku Corporation, Tokyo, Japan. Sheldrick, G. M. (2008). Acta Cryst. A64, 112-122. 


\section{supporting information}

Acta Cryst. (2015). E71, o1067-o1068 [https://doi.org/10.1107/S2056989015023907]

\section{Crystal structure of 3-bromo-9-ethyl-9H-carbazole}

\section{Mykola Bezuglyi, Gintare Grybauskaite, Gintautas Bagdziunas and Juozas Vidas Grazulevicius}

\section{S1. Synthesis and crystallization}

9-ethyl-carbazole $(1.00 \mathrm{~g}, 5.12 \mathrm{mmol})$ was added to a solution of N-bromosuccinimide $(0.911 \mathrm{~g}, 5.12 \mathrm{mmol})$ in $10 \mathrm{~mL}$ of DMF. The reaction mixture was refluxed at room temperature for 24 hours. When the reaction was completed (monitored via TLC) the solution was poured into a large amount of water with ice and extracted with ethyl acetate. The organic layer was dried over anhydrous sodium sulfate followed by solvent evaporation in rotary evaporator. The product was crystallized from methanol to afford a white needle-like crystals. Yield: $0.88 \mathrm{~g}(62 \%)$, melting point $58-60^{\circ} \mathrm{C} .{ }^{1} \mathrm{H}$ NMR $\left(700 \mathrm{MHz}, \mathrm{CDCl}_{3}\right) \delta 8.10(\mathrm{~d}, J=7.7 \mathrm{~Hz}, 1 \mathrm{H}), 7.63(\mathrm{~d}, J=2.5 \mathrm{~Hz}, 1 \mathrm{H}), 7.49(\mathrm{ddd}, J=8.2,7.1,1.1 \mathrm{~Hz}, 1 \mathrm{H}), 7.42(\mathrm{~d}, J=$ $8.2 \mathrm{~Hz}, 1 \mathrm{H}), 7.35(\mathrm{~d}, J=8.7 \mathrm{~Hz}, 1 \mathrm{H}), 7.25-7.22(\mathrm{~m}, 1 \mathrm{H}), 7.16(\mathrm{dd}, J=8.8,2.5 \mathrm{~Hz}, 1 \mathrm{H}), 4.37$ (q, $J=7.3 \mathrm{~Hz}, 2 \mathrm{H}), 1.45$ (t, $J=7.3 \mathrm{~Hz}, 4 \mathrm{H})$.

\section{S2. Refinement}

All hydrogen atoms were placed in geometrically idealized positions and constrained to ride on their parent atoms, with C $-\mathrm{H}=0.930 \AA$ for aromatic $\mathrm{C}-\mathrm{H}$, with $0.969 \AA$ for methylene $\mathrm{C}-\mathrm{H}, 0.957 \AA$ for methyl distances and $\mathrm{U}_{\text {iso }}(\mathrm{H})=1.2$ $\mathrm{U}_{\mathrm{eq}}$. 


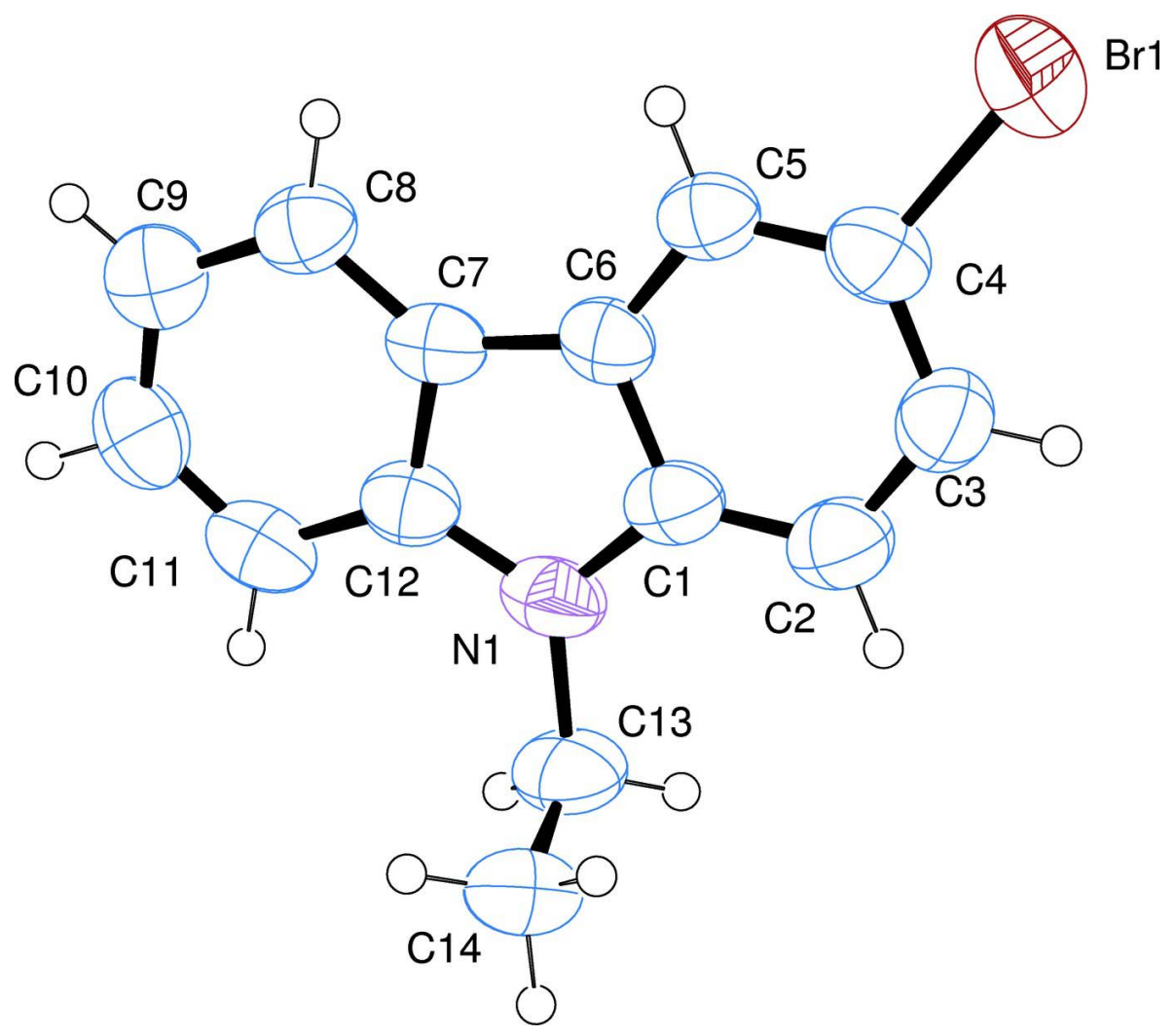

Figure 1

The molecular structure of the title molecule with displacement ellipsoids drawn at the $50 \%$ probability level.

3-Bromo-9-ethyl-9H-carbazole

Crystal data

$\mathrm{C}_{14} \mathrm{H}_{12} \mathrm{BrN}$

$M_{r}=274.16$

Orthorhombic, $\mathrm{Pbca}$

Hall symbol: -P $2 \mathrm{ac} 2 \mathrm{ab}$

$a=15.263(16) \AA$

$b=7.745(8) \AA$

$c=20.41(2) \AA$

$V=2413(5) \AA^{3}$

$Z=8$

Data collection

Rigaku XtaLAB mini diffractometer

Detector resolution: 13.653 pixels $\mathrm{mm}^{-1}$

$\omega$ scans

Absorption correction: multi-scan

(REQAB; Rigaku, 1998)

$T_{\min }=0.450, T_{\max }=0.763$

8316 measured reflections
$F(000)=1104.00$

$D_{\mathrm{x}}=1.509 \mathrm{Mg} \mathrm{m}^{-3}$

Mo $K \alpha$ radiation, $\lambda=0.71075 \AA$

Cell parameters from 3894 reflections

$\theta=3.1-27.5^{\circ}$

$\mu=3.39 \mathrm{~mm}^{-1}$

$T=273 \mathrm{~K}$

Chip, colorless

$0.40 \times 0.09 \times 0.08 \mathrm{~mm}$

2721 independent reflections

1383 reflections with $F^{2}>2.0 \sigma\left(F^{2}\right)$

$R_{\text {int }}=0.056$

$\theta_{\max }=27.5^{\circ}$

$h=-18 \rightarrow 18$

$k=-10 \rightarrow 7$

$l=-26 \rightarrow 21$ 


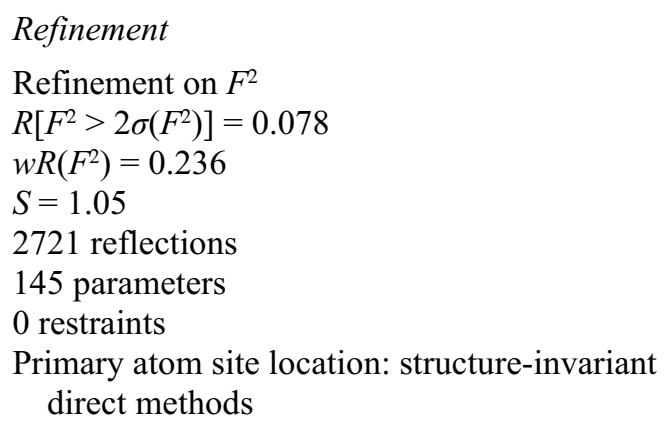

\section{Refinement}

Refinement on $F^{2}$

$R\left[F^{2}>2 \sigma\left(F^{2}\right)\right]=0.078$

2721 reflections

direct methods

Secondary atom site location: difference Fourier map

Hydrogen site location: inferred from neighbouring sites

$\mathrm{H}$-atom parameters constrained

$w=1 /\left[\sigma^{2}\left(F_{\mathrm{o}}^{2}\right)+(0.0926 P)^{2}+4.1576 P\right]$ where $P=\left(F_{\mathrm{o}}{ }^{2}+2 F_{\mathrm{c}}{ }^{2}\right) / 3$

$(\Delta / \sigma)_{\max }<0.001$

$\Delta \rho_{\max }=1.37 \mathrm{e} \AA^{-3}$

$\Delta \rho_{\min }=-0.46$ e $\AA^{-3}$

Special details

Geometry. ENTER SPECIAL DETAILS OF THE MOLECULAR GEOMETRY

Refinement. Refinement was performed using all reflections. The weighted R-factor (wR) and goodness of fit (S) are based on $\mathrm{F}^{2}$. R-factor (gt) are based on $\mathrm{F}$. The threshold expression of $\mathrm{F}^{2}>2.0 \operatorname{sigma}\left(\mathrm{F}^{2}\right)$ is used only for calculating Rfactor $(\mathrm{gt})$.

Fractional atomic coordinates and isotropic or equivalent isotropic displacement parameters $\left(\AA^{2}\right)$

\begin{tabular}{lllll}
\hline & $x$ & $y$ & $z$ & $U_{\text {iso }} * / U_{\text {eq }}$ \\
\hline Br1 & $0.28798(6)$ & $0.61687(12)$ & $0.45898(4)$ & $0.0841(5)$ \\
N1 & $0.6116(4)$ & $0.2371(7)$ & $0.3684(3)$ & $0.0635(15)$ \\
C1 & $0.5364(5)$ & $0.3073(8)$ & $0.3969(4)$ & $0.0570(17)$ \\
C2 & $0.4935(6)$ & $0.2633(10)$ & $0.4542(4)$ & $0.0680(19)$ \\
C3 & $0.4189(6)$ & $0.3557(10)$ & $0.4719(4)$ & $0.070(2)$ \\
C4 & $0.3891(5)$ & $0.4915(9)$ & $0.4327(4)$ & $0.0629(18)$ \\
C5 & $0.4317(5)$ & $0.5360(9)$ & $0.3759(4)$ & $0.0559(16)$ \\
C6 & $0.5065(5)$ & $0.4456(8)$ & $0.3573(4)$ & $0.0527(16)$ \\
C7 & $0.5643(4)$ & $0.4553(8)$ & $0.3016(4)$ & $0.0519(16)$ \\
C8 & $0.5686(5)$ & $0.5583(9)$ & $0.2455(4)$ & $0.0608(18)$ \\
C9 & $0.6333(5)$ & $0.5278(10)$ & $0.1987(4)$ & $0.071(2)$ \\
C10 & $0.6935(5)$ & $0.3978(11)$ & $0.2092(4)$ & $0.072(3)$ \\
C11 & $0.6939(5)$ & $0.2950(10)$ & $0.2632(5)$ & $0.067(2)$ \\
C12 & $0.6283(5)$ & $0.3243(9)$ & $0.3100(4)$ & $0.0577(17)$ \\
C13 & $0.6556(6)$ & $0.0790(9)$ & $0.3911(4)$ & $0.074(3)$ \\
C14 & $0.6145(6)$ & $-0.0826(9)$ & $0.3648(5)$ & $0.083(3)$ \\
H3 & 0.4107 & 0.6258 & 0.3500 & $0.0671^{*}$ \\
H8 & 0.7363 & 0.3795 & 0.1775 & $0.0866^{*}$ \\
H9 & 0.6358 & 0.5943 & 0.1608 & $0.0853^{*}$ \\
H10 & 0.5139 & 0.1737 & 0.4803 & $0.0816^{*}$ \\
H13 & 0.3888 & 0.3269 & 0.5100 & $0.0840^{*}$ \\
H14 & 0.5283 & 0.6470 & 0.2394 & $0.0730^{*}$ \\
H15 & 0.7358 & 0.2091 & 0.2688 & $0.0804^{*}$ \\
H17A & 0.6458 & -0.1813 & 0.3809 & $0.0995^{*}$ \\
H17B & 0.6167 & -0.0812 & 0.3178 & $0.0995^{*}$ \\
H17C & 0.5545 & -0.0887 & 0.3789 & $0.0995^{*}$ \\
H18A & 0.6540 & 0.0757 & 0.4386 & $0.0894^{*}$ \\
H18B & 0.7166 & 0.0827 & 0.3778 & $0.0894^{*}$ \\
& & & &
\end{tabular}


Atomic displacement parameters $\left(\AA^{2}\right)$

\begin{tabular}{lllllll}
\hline & $U^{11}$ & $U^{22}$ & $U^{33}$ & $U^{12}$ & $U^{13}$ & $U^{23}$ \\
\hline Br1 & $0.0778(7)$ & $0.0894(7)$ & $0.0852(7)$ & $0.0084(5)$ & $0.0147(5)$ & $-0.0107(5)$ \\
N1 & $0.067(4)$ & $0.053(3)$ & $0.071(4)$ & $0.017(3)$ & $-0.015(3)$ & $-0.007(3)$ \\
C1 & $0.071(5)$ & $0.043(4)$ & $0.056(4)$ & $-0.003(4)$ & $-0.017(4)$ & $-0.005(3)$ \\
C2 & $0.080(5)$ & $0.059(4)$ & $0.065(5)$ & $0.004(4)$ & $-0.012(4)$ & $0.004(4)$ \\
C3 & $0.091(6)$ & $0.063(5)$ & $0.056(5)$ & $-0.006(5)$ & $0.001(4)$ & $0.001(4)$ \\
C4 & $0.062(5)$ & $0.053(4)$ & $0.074(5)$ & $0.000(4)$ & $0.002(4)$ & $-0.008(4)$ \\
C5 & $0.061(4)$ & $0.045(4)$ & $0.061(4)$ & $-0.003(4)$ & $-0.010(4)$ & $-0.001(3)$ \\
C6 & $0.055(4)$ & $0.045(4)$ & $0.058(4)$ & $-0.003(3)$ & $-0.007(4)$ & $-0.009(3)$ \\
C7 & $0.049(4)$ & $0.043(4)$ & $0.063(4)$ & $-0.000(3)$ & $-0.017(3)$ & $-0.007(3)$ \\
C8 & $0.062(4)$ & $0.056(4)$ & $0.065(5)$ & $-0.001(4)$ & $-0.011(4)$ & $0.003(4)$ \\
C9 & $0.069(5)$ & $0.069(5)$ & $0.075(5)$ & $-0.011(5)$ & $-0.001(4)$ & $-0.005(4)$ \\
C10 & $0.064(5)$ & $0.083(6)$ & $0.070(5)$ & $-0.006(5)$ & $-0.002(4)$ & $-0.021(5)$ \\
C11 & $0.054(4)$ & $0.060(5)$ & $0.087(6)$ & $0.007(4)$ & $-0.014(4)$ & $-0.019(4)$ \\
C12 & $0.054(4)$ & $0.050(4)$ & $0.068(5)$ & $0.001(4)$ & $-0.007(4)$ & $-0.004(4)$ \\
C13 & $0.080(5)$ & $0.064(5)$ & $0.080(5)$ & $0.020(4)$ & $-0.023(5)$ & $0.007(4)$ \\
C14 & $0.104(7)$ & $0.051(4)$ & $0.094(6)$ & $0.010(5)$ & $0.001(5)$ & $0.003(4)$ \\
& & & & & & \\
\hline
\end{tabular}

Geometric parameters $\left(\AA,{ }^{\circ}\right)$

\begin{tabular}{|c|c|c|c|}
\hline $\mathrm{Br} 1-\mathrm{C} 4$ & $1.901(7)$ & $\mathrm{C} 10-\mathrm{C} 11$ & $1.358(12)$ \\
\hline $\mathrm{N} 1-\mathrm{C} 1$ & $1.396(9)$ & $\mathrm{C} 11-\mathrm{C} 12$ & $1.403(11)$ \\
\hline $\mathrm{N} 1-\mathrm{C} 12$ & $1.393(10)$ & $\mathrm{C} 13-\mathrm{C} 14$ & $1.499(11)$ \\
\hline $\mathrm{N} 1-\mathrm{C} 13$ & $1.471(10)$ & $\mathrm{C} 2-\mathrm{H} 10$ & 0.930 \\
\hline $\mathrm{C} 1-\mathrm{C} 2$ & $1.382(10)$ & $\mathrm{C} 3-\mathrm{H} 13$ & 0.930 \\
\hline $\mathrm{C} 1-\mathrm{C} 6$ & $1.418(9)$ & $\mathrm{C} 5-\mathrm{H} 3$ & 0.930 \\
\hline $\mathrm{C} 2-\mathrm{C} 3$ & $1.392(12)$ & $\mathrm{C} 8-\mathrm{H} 14$ & 0.930 \\
\hline $\mathrm{C} 3-\mathrm{C} 4$ & $1.397(11)$ & $\mathrm{C} 9-\mathrm{H} 9$ & 0.930 \\
\hline $\mathrm{C} 4-\mathrm{C} 5$ & $1.373(10)$ & $\mathrm{C} 10-\mathrm{H} 8$ & 0.930 \\
\hline $\mathrm{C} 5-\mathrm{C} 6$ & $1.391(9)$ & C11-H15 & 0.930 \\
\hline $\mathrm{C} 6-\mathrm{C} 7$ & $1.442(10)$ & C13-H18A & 0.970 \\
\hline $\mathrm{C} 7-\mathrm{C} 8$ & $1.397(10)$ & C13-H18B & 0.970 \\
\hline $\mathrm{C} 7-\mathrm{C} 12$ & $1.419(9)$ & $\mathrm{C} 14-\mathrm{H} 17 \mathrm{~A}$ & 0.960 \\
\hline $\mathrm{C} 8-\mathrm{C} 9$ & $1.394(11)$ & C14-H17B & 0.960 \\
\hline $\mathrm{C} 9-\mathrm{C} 10$ & $1.381(11)$ & $\mathrm{C} 14-\mathrm{H} 17 \mathrm{C}$ & 0.960 \\
\hline $\mathrm{N} 1 \cdots \mathrm{C} 5$ & $3.594(9)$ & $\mathrm{C} 10 \cdots \mathrm{H} 3^{\mathrm{vi}}$ & 2.9041 \\
\hline $\mathrm{N} 1 \cdots \mathrm{C} 8$ & $3.594(10)$ & $\mathrm{C} 10 \cdots \mathrm{H} 15^{\text {ix }}$ & 2.9077 \\
\hline $\mathrm{C} 1 \cdots \mathrm{C} 4$ & $2.761(10)$ & $\mathrm{C} 11 \cdots \mathrm{H} 3^{\mathrm{vi}}$ & 3.1000 \\
\hline $\mathrm{C} 1 \cdots \mathrm{C} 14$ & $3.311(11)$ & $\mathrm{C} 11 \cdots \mathrm{H} 14^{\mathrm{vi}}$ & 3.5811 \\
\hline $\mathrm{C} 2 \cdots \mathrm{C} 5$ & $2.811(10)$ & $\mathrm{C} 11 \cdots \mathrm{H} 15^{\mathrm{ix}}$ & 3.3834 \\
\hline $\mathrm{C} 2 \cdots \mathrm{C} 13$ & $3.133(12)$ & $\mathrm{C} 11 \cdots \mathrm{H} 17 \mathrm{~A}^{\mathrm{ix}}$ & 3.4343 \\
\hline $\mathrm{C} 3 \cdots \mathrm{C} 6$ & $2.781(11)$ & $\mathrm{C} 11 \cdots \mathrm{H} 17 \mathrm{~B}^{\mathrm{ix}}$ & 3.2429 \\
\hline $\mathrm{C} 5 \cdots \mathrm{C} 8$ & $3.388(10)$ & $\mathrm{C} 11 \cdots \mathrm{H} 18 \mathrm{~B}^{\mathrm{ix}}$ & 3.5072 \\
\hline $\mathrm{C} 7 \cdots \mathrm{C} 10$ & $2.764(11)$ & $\mathrm{C} 12 \cdots \mathrm{H} 14^{\mathrm{vi}}$ & 2.9363 \\
\hline $\mathrm{C} 8 \cdots \mathrm{C} 11$ & $2.819(10)$ & $\mathrm{C} 12 \cdots \mathrm{H} 18 \mathrm{~B}^{\mathrm{ix}}$ & 3.3937 \\
\hline
\end{tabular}




\begin{tabular}{|c|c|c|c|}
\hline $\mathrm{C} 9 \cdots \mathrm{C} 12$ & 2.767 (11) & $\mathrm{C} 13 \cdots \mathrm{H} 17 \mathrm{~A}^{\mathrm{ix}}$ & 3.5602 \\
\hline $\mathrm{C} 11 \cdots \mathrm{C} 13$ & $3.156(12)$ & $\mathrm{C} 14 \cdots \mathrm{H} 13^{\mathrm{vii}}$ & 3.1805 \\
\hline $\mathrm{C} 12 \cdots \mathrm{C} 14$ & $3.351(11)$ & $\mathrm{C} 14 \cdots \mathrm{H} 14^{\mathrm{x}}$ & 3.5604 \\
\hline$C 2 \cdots C 3^{i}$ & $3.574(11)$ & $\mathrm{C} 14 \cdots \mathrm{H} 14^{\mathrm{vi}}$ & 3.5260 \\
\hline $\mathrm{C} 2 \cdots \mathrm{C} 4^{\mathrm{i}}$ & $3.486(11)$ & $\mathrm{C} 14 \cdots \mathrm{H} 15^{\mathrm{xi}}$ & 3.4162 \\
\hline $\mathrm{C} 3 \cdots \mathrm{C} 2^{\mathrm{i}}$ & $3.574(11)$ & $\mathrm{H} 3 \cdots \mathrm{C} 9^{\mathrm{ii}}$ & 3.3372 \\
\hline $\mathrm{C} 3 \cdots \mathrm{C} 3^{\mathrm{i}}$ & $3.527(12)$ & $\mathrm{H} 3 \cdots \mathrm{C} 10^{\mathrm{ii}}$ & 2.9041 \\
\hline $\mathrm{C} 4 \cdots \mathrm{C} 2^{\mathrm{i}}$ & $3.486(11)$ & $\mathrm{H} 3 \cdots \mathrm{C} 11^{\mathrm{ii}}$ & 3.1000 \\
\hline $\mathrm{Br} 1 \cdots \mathrm{H} 3$ & 2.9084 & $\mathrm{H} 3 \cdots \mathrm{H} 8^{\mathrm{ii}}$ & 3.0356 \\
\hline $\mathrm{Br} 1 \cdots \mathrm{H} 13$ & 2.9144 & $\mathrm{H} 3 \cdots \mathrm{H}^{\mathrm{iii}}$ & 3.3234 \\
\hline $\mathrm{N} 1 \cdots \mathrm{H} 10$ & 2.7717 & $\mathrm{H} 3 \cdots \mathrm{H} 15^{\mathrm{ii}}$ & 3.3615 \\
\hline $\mathrm{N} 1 \cdots \mathrm{H} 15$ & 2.7889 & $\mathrm{H} 3 \cdots \mathrm{H} 17 \mathrm{C}^{\text {viii }}$ & 3.1708 \\
\hline 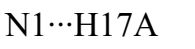 & 3.2924 & $\mathrm{H} 8 \cdots \mathrm{Br}^{\mathrm{vi}}$ & 3.4695 \\
\hline $\mathrm{N} 1 \cdots \mathrm{H} 17 \mathrm{~B}$ & 2.6741 & $\mathrm{H} 8 \cdots \mathrm{Br} 1^{\mathrm{xii}}$ & 3.4304 \\
\hline $\mathrm{N} 1 \cdots \mathrm{H} 17 \mathrm{C}$ & 2.6783 & $\mathrm{H} 8 \cdots \mathrm{C}^{\mathrm{xii}}$ & 3.3551 \\
\hline $\mathrm{C} 1 \cdots \mathrm{H} 3$ & 3.2683 & $\mathrm{H} 8 \cdots \mathrm{C}^{\mathrm{xii}}$ & 3.3993 \\
\hline $\mathrm{C} 1 \cdots \mathrm{H} 13$ & 3.2280 & $\mathrm{H} 8 \cdots \mathrm{C} 9^{\mathrm{xi}}$ & 3.4012 \\
\hline $\mathrm{C} 1 \cdots \mathrm{H} 17 \mathrm{C}$ & 3.1012 & $\mathrm{H} 8 \cdots \mathrm{H}^{\mathrm{vi}}$ & 3.0356 \\
\hline $\mathrm{C} 1 \cdots \mathrm{H} 18 \mathrm{~A}$ & 2.6759 & $\mathrm{H} 8 \cdots \mathrm{H} 3^{\mathrm{xii}}$ & 3.3234 \\
\hline $\mathrm{C} 1 \cdots \mathrm{H} 18 \mathrm{~B}$ & 3.2769 & $\mathrm{H} 8 \cdots{ }^{\prime} 9^{\mathrm{xi}}$ & 2.9669 \\
\hline $\mathrm{C} 2 \cdots \mathrm{H} 17 \mathrm{C}$ & 3.2655 & $\mathrm{H} 8 \cdots \mathrm{H} 15^{\text {ix }}$ & 3.1884 \\
\hline $\mathrm{C} 2 \cdots \mathrm{H} 18 \mathrm{~A}$ & 2.8668 & $\mathrm{H} 9 \cdots \mathrm{Br} 1^{\mathrm{xii}}$ & 3.3766 \\
\hline $\mathrm{C} 3 \cdots \mathrm{H} 3$ & 3.2516 & $\mathrm{H} 9 \cdots \mathrm{C} 1^{\mathrm{ii}}$ & 3.3199 \\
\hline $\mathrm{C} 4 \cdots \mathrm{H} 10$ & 3.2612 & $\mathrm{H} 9 \cdots \mathrm{C} 2^{\mathrm{ii}}$ & 3.3337 \\
\hline $\mathrm{C} 5 \cdots \mathrm{H} 13$ & 3.2457 & $\mathrm{H} 9 \cdots \mathrm{C} 3^{\mathrm{ii}}$ & 3.4827 \\
\hline $\mathrm{C} 5 \cdots \mathrm{H} 14$ & 3.2684 & $\mathrm{H} 9 \cdots \mathrm{C}^{\mathrm{ii}}$ & 3.5009 \\
\hline C6 $\cdots \mathrm{H} 10$ & 3.2790 & $\mathrm{H} 9 \cdots \mathrm{H} 8^{\mathrm{ix}}$ & 2.9669 \\
\hline C6 $\cdots \mathrm{H} 14$ & 2.8883 & $\mathrm{H} 9 \cdots \mathrm{H} 15^{\text {ix }}$ & 3.0795 \\
\hline $\mathrm{C} 7 \cdots \mathrm{H} 3$ & 2.8662 & $\mathrm{H} 9 \cdots \mathrm{H} 17 \mathrm{C}^{\mathrm{ii}}$ & 3.3331 \\
\hline $\mathrm{C} 7 \cdots \mathrm{H} 9$ & 3.2568 & $\mathrm{H} 10 \cdots \mathrm{C} 4^{\mathrm{i}}$ & 3.4733 \\
\hline $\mathrm{C} 7 \cdots \mathrm{H} 15$ & 3.3072 & $\mathrm{H} 10 \cdots \mathrm{H} 10^{\mathrm{vii}}$ & 2.8396 \\
\hline $\mathrm{C} 8 \cdots \mathrm{H} 3$ & 3.2607 & $\mathrm{H} 10 \cdots \mathrm{H} 17 \mathrm{C}^{\mathrm{vii}}$ & 3.1274 \\
\hline $\mathrm{C} 8 \cdots \mathrm{H} 8$ & 3.2241 & $\mathrm{H} 13 \cdots \mathrm{Br} 1^{\mathrm{xiii}}$ & 3.3182 \\
\hline C9 $\cdots \mathrm{H} 15$ & 3.2540 & $\mathrm{H} 13 \cdots \mathrm{C}^{\mathrm{i}}$ & 3.5975 \\
\hline $\mathrm{C} 10 \cdots \mathrm{H} 14$ & 3.2341 & $\mathrm{H} 13 \cdots \mathrm{C} 14^{\mathrm{vii}}$ & 3.1805 \\
\hline $\mathrm{C} 11 \cdots \mathrm{H} 9$ & 3.2440 & $\mathrm{H} 13 \cdots \mathrm{H} 17 \mathrm{~A}^{\mathrm{vii}}$ & 2.5518 \\
\hline C11H17B & 3.3353 & $\mathrm{H} 13 \cdots \mathrm{H} 17 \mathrm{C}^{\mathrm{vii}}$ & 3.0494 \\
\hline 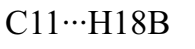 & 2.8803 & $\mathrm{H} 13 \cdots \mathrm{H} 18 \mathrm{~A}^{\mathrm{vii}}$ & 3.3550 \\
\hline $\mathrm{C} 12 \cdots \mathrm{H} 8$ & 3.1954 & $\mathrm{H} 13 \cdots \mathrm{H} 18 \mathrm{~B}^{\mathrm{v}}$ & 3.5574 \\
\hline $\mathrm{C} 12 \cdots \mathrm{H} 14$ & 3.2644 & $\mathrm{H} 14 \cdots \mathrm{N} 1^{\mathrm{ii}}$ & 3.1441 \\
\hline $\mathrm{C} 12 \cdots \mathrm{H} 17 \mathrm{~B}$ & 3.1500 & $\mathrm{H} 14 \cdots \mathrm{C} 1^{\mathrm{ii}}$ & 3.2025 \\
\hline $\mathrm{C} 12 \cdots \mathrm{H} 18 \mathrm{~A}$ & 3.2783 & $\mathrm{H} 14 \cdots \mathrm{C}^{\mathrm{ii}}$ & 3.0863 \\
\hline $\mathrm{C} 12 \cdots \mathrm{H} 18 \mathrm{~B}$ & 2.6882 & $\mathrm{H} 14 \cdots \mathrm{C} 7^{\mathrm{ii}}$ & 2.8977 \\
\hline $\mathrm{C} 13 \cdots \mathrm{H} 10$ & 2.9205 & $\mathrm{H} 14 \cdots \mathrm{C}^{\mathrm{ii}}$ & 3.5256 \\
\hline $\mathrm{C} 13 \cdots \mathrm{H} 15$ & 2.9579 & $\mathrm{H} 14 \cdots \mathrm{C} 11^{\mathrm{ii}}$ & 3.5811 \\
\hline $\mathrm{C} 14 \cdots \mathrm{H} 10$ & 3.4427 & $\mathrm{H} 14 \cdots \mathrm{C} 12^{\mathrm{ii}}$ & 2.9363 \\
\hline $\mathrm{C} 14 \cdots \mathrm{H} 15$ & 3.5181 & $\mathrm{H} 14 \cdots \mathrm{C} 14^{\text {viii }}$ & 3.5604 \\
\hline H3 $\cdots \mathrm{H} 14$ & 2.8901 & $\mathrm{H} 14 \cdots \mathrm{C} 14^{\mathrm{ii}}$ & 3.5260 \\
\hline
\end{tabular}




\begin{tabular}{|c|c|c|c|}
\hline $\mathrm{H} 8 \cdots \mathrm{H} 9$ & 2.2887 & $\mathrm{H} 14 \cdots \mathrm{H} 17 \mathrm{~B}^{\mathrm{viii}}$ & 2.9697 \\
\hline H8 $\cdots H 15$ & 2.2820 & $\mathrm{H} 14 \cdots \mathrm{H} 17 \mathrm{~B}^{\mathrm{ii}}$ & 3.0636 \\
\hline H9 $\cdots$ H14 & 2.3299 & H14 $\cdots H 17 C^{\text {viii }}$ & 3.5302 \\
\hline $\mathrm{H} 10 \cdots \mathrm{H} 13$ & 2.3284 & $\mathrm{H} 14 \cdots \mathrm{H} 17 \mathrm{C}^{\mathrm{ii}}$ & 3.2796 \\
\hline $\mathrm{H} 10 \cdots \mathrm{H} 17 \mathrm{C}$ & 2.9668 & $\mathrm{H} 15 \cdots \mathrm{C} 8^{\mathrm{xi}}$ & 3.2416 \\
\hline $\mathrm{H} 10 \cdots \mathrm{H} 18 \mathrm{~A}$ & 2.4234 & $\mathrm{H} 15 \cdots \mathrm{C}^{\mathrm{xi}}$ & 2.8304 \\
\hline 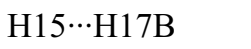 & 3.0595 & $\mathrm{H} 15 \cdots \mathrm{C} 10^{\mathrm{xi}}$ & 2.9077 \\
\hline 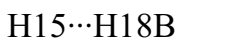 & 2.4483 & $\mathrm{H} 15 \cdots \mathrm{C} 11^{\mathrm{xi}}$ & 3.3834 \\
\hline $\mathrm{H} 17 \mathrm{~A} \cdots \mathrm{H} 18 \mathrm{~A}$ & 2.3159 & $\mathrm{H} 15 \cdots \mathrm{C} 14^{\mathrm{ix}}$ & 3.4162 \\
\hline H17A $\cdots H 18 B$ & 2.3126 & $\mathrm{H} 15 \cdots \mathrm{H} 3^{\mathrm{vi}}$ & 3.3615 \\
\hline H17B $\cdots H 18 A$ & 2.8071 & $\mathrm{H} 15 \cdots \mathrm{H} 8^{\mathrm{xi}}$ & 3.1884 \\
\hline $\mathrm{H} 17 \mathrm{~B} \cdots \mathrm{H} 18 \mathrm{~B}$ & 2.3301 & $\mathrm{H} 15 \cdots \mathrm{H} 9^{\mathrm{xi}}$ & 3.0795 \\
\hline $\mathrm{H} 17 \mathrm{C} \cdots \mathrm{H} 18 \mathrm{~A}$ & 2.3268 & $\mathrm{H} 15 \cdots \mathrm{H} 17 \mathrm{~A}^{\mathrm{ix}}$ & 3.0376 \\
\hline $\mathrm{H} 17 \mathrm{C} \cdots \mathrm{H} 18 \mathrm{~B}$ & 2.8068 & $\mathrm{H} 15 \cdots \mathrm{H} 17 \mathrm{~B}^{\mathrm{ix}}$ & 2.9508 \\
\hline $\mathrm{Br} 1 \cdots \mathrm{H} 8^{\mathrm{ii}}$ & 3.4695 & $\mathrm{H} 17 \mathrm{~A} \cdots \mathrm{C} 3^{\mathrm{vii}}$ & 3.4391 \\
\hline $\mathrm{Br} 1 \cdots \mathrm{H} 8^{\mathrm{iii}}$ & 3.4304 & $\mathrm{H} 17 \mathrm{~A}^{\cdots} \cdot \mathrm{C}^{\mathrm{x}}$ & 3.4783 \\
\hline $\mathrm{Br} 1 \cdots \mathrm{H} 9^{\mathrm{iii}}$ & 3.3766 & $\mathrm{H} 17 \mathrm{~A} \cdots \mathrm{C} 11^{\mathrm{xi}}$ & 3.4343 \\
\hline $\mathrm{Br} 1 \cdots \mathrm{H} 13^{\text {iv }}$ & 3.3182 & $\mathrm{H} 17 \mathrm{~A} \cdots \mathrm{C} 13^{\mathrm{xi}}$ & 3.5602 \\
\hline $\mathrm{Br} 1 \cdots \mathrm{H} 18 \mathrm{~A}^{\mathrm{i}}$ & 3.2895 & H17A $\cdots H 13^{\text {vii }}$ & 2.5518 \\
\hline $\mathrm{Br} 1 \cdots \mathrm{H} 18 \mathrm{~A}^{\mathrm{v}}$ & 3.2825 & $\mathrm{H} 17 \mathrm{~A} \cdots \mathrm{H} 15^{\mathrm{xi}}$ & 3.0376 \\
\hline $\mathrm{N} 1 \cdots \mathrm{H} 14^{\mathrm{vi}}$ & 3.1441 & $\mathrm{H} 17 \mathrm{~A} \cdots \mathrm{H} 18 \mathrm{~B}^{\mathrm{xi}}$ & 2.7853 \\
\hline $\mathrm{C} 1 \cdots \mathrm{H} 9^{\mathrm{vi}}$ & 3.3199 & $\mathrm{H} 17 \mathrm{~B} \cdots \mathrm{C} 8^{\mathrm{x}}$ & 3.2433 \\
\hline $\mathrm{C} 1 \cdots \mathrm{H} 13^{\mathrm{i}}$ & 3.5975 & $\mathrm{H} 17 \mathrm{~B} \cdots \mathrm{C} 8^{\mathrm{vi}}$ & 3.2915 \\
\hline $\mathrm{C} 1 \cdots \mathrm{H} 14^{\mathrm{vi}}$ & 3.2025 & $\mathrm{H} 17 \mathrm{~B} \cdots \mathrm{C} 11^{\mathrm{xi}}$ & 3.2429 \\
\hline $\mathrm{C} 2 \cdots \mathrm{H} 9^{\mathrm{vi}}$ & 3.3337 & H17B $\cdots H 14^{x}$ & 2.9697 \\
\hline $\mathrm{C} 3 \cdots \mathrm{H} 9^{\text {vi }}$ & 3.4827 & H17B $\cdots H 14^{\mathrm{vi}}$ & 3.0636 \\
\hline $\mathrm{C} 3 \cdots \mathrm{H} 17 \mathrm{~A}^{\mathrm{vii}}$ & 3.4391 & $\mathrm{H} 17 \mathrm{~B} \cdots \mathrm{H} 15^{\mathrm{xi}}$ & 2.9508 \\
\hline $\mathrm{C} 4 \cdots \mathrm{H}^{\mathrm{iii}}$ & 3.3551 & $\mathrm{H} 17 \mathrm{C} \cdots \mathrm{C} 5^{\mathrm{x}}$ & 3.4596 \\
\hline $\mathrm{C} 4 \cdots \mathrm{H} 10^{\mathrm{i}}$ & 3.4733 & $\mathrm{H} 17 \mathrm{C} \cdots \mathrm{C} 8^{\mathrm{vi}}$ & 3.3569 \\
\hline $\mathrm{C} 5 \cdots \mathrm{H} 8^{\mathrm{iii}}$ & 3.3993 & $\mathrm{H} 17 \mathrm{C} \cdots \mathrm{C} 9^{\mathrm{vi}}$ & 3.3968 \\
\hline $\mathrm{C} 5 \cdots \mathrm{H} 17 \mathrm{C}^{\mathrm{viii}}$ & 3.4596 & $\mathrm{H} 17 \mathrm{C} \cdots \mathrm{H} 3^{\mathrm{x}}$ & 3.1708 \\
\hline $\mathrm{C} 6 \cdots \mathrm{H}^{\mathrm{vi}}$ & 3.5009 & $\mathrm{H} 17 \mathrm{C} \cdots \mathrm{H} 9^{\text {vi }}$ & 3.3331 \\
\hline $\mathrm{C} 6 \cdots \mathrm{H} 14^{\mathrm{vi}}$ & 3.0863 & $\mathrm{H} 17 \mathrm{C} \cdots \mathrm{H} 10^{\text {vii }}$ & 3.1274 \\
\hline $\mathrm{C} 7 \cdots \mathrm{H} 14^{\mathrm{vi}}$ & 2.8977 & $\mathrm{H} 17 \mathrm{C} \cdots \mathrm{H} 13^{\mathrm{vii}}$ & 3.0494 \\
\hline $\mathrm{C} 7 \cdots \mathrm{H} 17 \mathrm{~A}^{\mathrm{viii}}$ & 3.4783 & $\mathrm{H} 17 \mathrm{C} \cdots \mathrm{H} 14^{\mathrm{x}}$ & 3.5302 \\
\hline $\mathrm{C} 8 \cdots \mathrm{H} 14^{\mathrm{vi}}$ & 3.5256 & $\mathrm{H} 17 \mathrm{C} \cdots \mathrm{H} 14^{\mathrm{vi}}$ & 3.2796 \\
\hline $\mathrm{C} 8 \cdots \mathrm{H} 15^{\mathrm{ix}}$ & 3.2416 & $\mathrm{H} 18 \mathrm{~A} \cdots \mathrm{Br} 1^{\mathrm{i}}$ & 3.2895 \\
\hline $\mathrm{C} 8 \cdots \mathrm{H} 17 \mathrm{~B}^{\mathrm{viii}}$ & 3.2433 & $\mathrm{H} 18 \mathrm{~A} \cdots \mathrm{Br} 1^{\text {xiv }}$ & 3.2825 \\
\hline $\mathrm{C} 8 \cdots \mathrm{H} 17 \mathrm{~B}^{\mathrm{ii}}$ & 3.2915 & H18A $\cdots$ H13 $3^{\text {vii }}$ & 3.3550 \\
\hline $\mathrm{C} 8 \cdots \mathrm{H} 17 \mathrm{C}^{\mathrm{ii}}$ & 3.3569 & $\mathrm{H} 18 \mathrm{~B} \cdots \mathrm{C} 11^{\mathrm{xi}}$ & 3.5072 \\
\hline $\mathrm{C} 9 \cdots \mathrm{H} 3^{\mathrm{vi}}$ & 3.3372 & $\mathrm{H} 18 \mathrm{~B} \cdots \mathrm{C} 12^{\mathrm{xi}}$ & 3.3937 \\
\hline C $9 \cdots H 8^{\text {ix }}$ & 3.4012 & $\mathrm{H} 18 \mathrm{~B} \cdots \mathrm{H} 13^{\mathrm{xiv}}$ & 3.5574 \\
\hline $\mathrm{C} 9 \cdots \mathrm{H} 15^{\text {ix }}$ & 2.8304 & $\mathrm{H} 18 \mathrm{~B} \cdots \mathrm{H} 17 \mathrm{~A}^{\mathrm{ix}}$ & 2.7853 \\
\hline $\mathrm{C} 9 \cdots \mathrm{H} 17 \mathrm{C}^{\mathrm{ii}}$ & 3.3968 & & \\
\hline $\mathrm{C} 1-\mathrm{N} 1-\mathrm{C} 12$ & $108.6(6)$ & $\mathrm{C} 1-\mathrm{C} 2-\mathrm{H} 10$ & 120.636 \\
\hline $\mathrm{C} 1-\mathrm{N} 1-\mathrm{C} 13$ & $124.6(6)$ & $\mathrm{C} 3-\mathrm{C} 2-\mathrm{H} 10$ & 120.638 \\
\hline $\mathrm{C} 12-\mathrm{N} 1-\mathrm{C} 13$ & $126.1(6)$ & $\mathrm{C} 2-\mathrm{C} 3-\mathrm{H} 13$ & 119.841 \\
\hline
\end{tabular}




\begin{tabular}{|c|c|c|c|}
\hline $\mathrm{N} 1-\mathrm{C} 1-\mathrm{C} 2$ & $130.3(7)$ & $\mathrm{C} 4-\mathrm{C} 3-\mathrm{H} 13$ & 119.848 \\
\hline $\mathrm{N} 1-\mathrm{C} 1-\mathrm{C} 6$ & $108.7(6)$ & $\mathrm{C} 4-\mathrm{C} 5-\mathrm{H} 3$ & 120.246 \\
\hline $\mathrm{C} 2-\mathrm{C} 1-\mathrm{C} 6$ & $121.0(7)$ & $\mathrm{C} 6-\mathrm{C} 5-\mathrm{H} 3$ & 120.252 \\
\hline $\mathrm{C} 1-\mathrm{C} 2-\mathrm{C} 3$ & $118.7(7)$ & $\mathrm{C} 7-\mathrm{C} 8-\mathrm{H} 14$ & 120.069 \\
\hline $\mathrm{C} 2-\mathrm{C} 3-\mathrm{C} 4$ & $120.3(7)$ & $\mathrm{C} 9-\mathrm{C} 8-\mathrm{H} 14$ & 120.061 \\
\hline $\mathrm{Br} 1-\mathrm{C} 4-\mathrm{C} 3$ & $119.2(6)$ & $\mathrm{C} 8-\mathrm{C} 9-\mathrm{H} 9$ & 120.379 \\
\hline $\mathrm{Br} 1-\mathrm{C} 4-\mathrm{C} 5$ & $119.7(6)$ & $\mathrm{C} 10-\mathrm{C} 9-\mathrm{H} 9$ & 120.368 \\
\hline $\mathrm{C} 3-\mathrm{C} 4-\mathrm{C} 5$ & $121.2(7)$ & $\mathrm{C} 9-\mathrm{C} 10-\mathrm{H} 8$ & 118.092 \\
\hline $\mathrm{C} 4-\mathrm{C} 5-\mathrm{C} 6$ & $119.5(6)$ & $\mathrm{C} 11-\mathrm{C} 10-\mathrm{H} 8$ & 118.084 \\
\hline $\mathrm{C} 1-\mathrm{C} 6-\mathrm{C} 5$ & $119.3(6)$ & $\mathrm{C} 10-\mathrm{C} 11-\mathrm{H} 15$ & 121.478 \\
\hline $\mathrm{C} 1-\mathrm{C} 6-\mathrm{C} 7$ & $107.0(6)$ & $\mathrm{C} 12-\mathrm{C} 11-\mathrm{H} 15$ & 121.484 \\
\hline $\mathrm{C} 5-\mathrm{C} 6-\mathrm{C} 7$ & $133.7(6)$ & $\mathrm{N} 1-\mathrm{C} 13-\mathrm{H} 18 \mathrm{~A}$ & 108.980 \\
\hline $\mathrm{C} 6-\mathrm{C} 7-\mathrm{C} 8$ & $134.8(6)$ & $\mathrm{N} 1-\mathrm{C} 13-\mathrm{H} 18 \mathrm{~B}$ & 108.983 \\
\hline $\mathrm{C} 6-\mathrm{C} 7-\mathrm{C} 12$ & $106.8(6)$ & $\mathrm{C} 14-\mathrm{C} 13-\mathrm{H} 18 \mathrm{~A}$ & 108.979 \\
\hline $\mathrm{C} 8-\mathrm{C} 7-\mathrm{C} 12$ & $118.4(6)$ & $\mathrm{C} 14-\mathrm{C} 13-\mathrm{H} 18 \mathrm{~B}$ & 108.984 \\
\hline $\mathrm{C} 7-\mathrm{C} 8-\mathrm{C} 9$ & $119.9(7)$ & $\mathrm{H} 18 \mathrm{~A}-\mathrm{C} 13-\mathrm{H} 18 \mathrm{~B}$ & 107.770 \\
\hline $\mathrm{C} 8-\mathrm{C} 9-\mathrm{C} 10$ & $119.3(8)$ & $\mathrm{C} 13-\mathrm{C} 14-\mathrm{H} 17 \mathrm{~A}$ & 109.478 \\
\hline $\mathrm{C} 9-\mathrm{C} 10-\mathrm{C} 11$ & $123.8(8)$ & $\mathrm{C} 13-\mathrm{C} 14-\mathrm{H} 17 \mathrm{~B}$ & 109.463 \\
\hline $\mathrm{C} 10-\mathrm{C} 11-\mathrm{C} 12$ & $117.0(7)$ & $\mathrm{C} 13-\mathrm{C} 14-\mathrm{H} 17 \mathrm{C}$ & 109.470 \\
\hline $\mathrm{N} 1-\mathrm{C} 12-\mathrm{C} 7$ & $108.9(6)$ & $\mathrm{H} 17 \mathrm{~A}-\mathrm{C} 14-\mathrm{H} 17 \mathrm{~B}$ & 109.470 \\
\hline $\mathrm{N} 1-\mathrm{C} 12-\mathrm{C} 11$ & $129.5(7)$ & $\mathrm{H} 17 \mathrm{~A}-\mathrm{C} 14-\mathrm{H} 17 \mathrm{C}$ & 109.473 \\
\hline $\mathrm{C} 7-\mathrm{C} 12-\mathrm{C} 11$ & $121.6(7)$ & $\mathrm{H} 17 \mathrm{~B}-\mathrm{C} 14-\mathrm{H} 17 \mathrm{C}$ & 109.472 \\
\hline $\mathrm{N} 1-\mathrm{C} 13-\mathrm{C} 14$ & $113.0(7)$ & & \\
\hline $\mathrm{C} 1-\mathrm{N} 1-\mathrm{C} 12-\mathrm{C} 7$ & $1.4(7)$ & $\mathrm{Br} 1-\mathrm{C} 4-\mathrm{C} 5-\mathrm{C} 6$ & $179.0(4)$ \\
\hline $\mathrm{C} 1-\mathrm{N} 1-\mathrm{C} 12-\mathrm{C} 11$ & $-176.9(6)$ & $\mathrm{C} 3-\mathrm{C} 4-\mathrm{C} 5-\mathrm{C} 6$ & $-0.8(10)$ \\
\hline $\mathrm{C} 12-\mathrm{N} 1-\mathrm{C} 1-\mathrm{C} 2$ & $179.1(6)$ & $\mathrm{C} 4-\mathrm{C} 5-\mathrm{C} 6-\mathrm{C} 1$ & $1.0(9)$ \\
\hline $\mathrm{C} 12-\mathrm{N} 1-\mathrm{C} 1-\mathrm{C} 6$ & $-2.1(7)$ & $\mathrm{C} 4-\mathrm{C} 5-\mathrm{C} 6-\mathrm{C} 7$ & $178.2(6)$ \\
\hline $\mathrm{C} 1-\mathrm{N} 1-\mathrm{C} 13-\mathrm{C} 14$ & $83.0(8)$ & $\mathrm{C} 1-\mathrm{C} 6-\mathrm{C} 7-\mathrm{C} 8$ & $178.2(6)$ \\
\hline $\mathrm{C} 13-\mathrm{N} 1-\mathrm{C} 1-\mathrm{C} 2$ & $8.6(11)$ & $\mathrm{C} 1-\mathrm{C} 6-\mathrm{C} 7-\mathrm{C} 12$ & $-1.1(7)$ \\
\hline $\mathrm{C} 13-\mathrm{N} 1-\mathrm{C} 1-\mathrm{C} 6$ & $-172.6(6)$ & $\mathrm{C} 5-\mathrm{C} 6-\mathrm{C} 7-\mathrm{C} 8$ & $0.7(13)$ \\
\hline $\mathrm{C} 12-\mathrm{N} 1-\mathrm{C} 13-\mathrm{C} 14$ & $-85.8(8)$ & $\mathrm{C} 5-\mathrm{C} 6-\mathrm{C} 7-\mathrm{C} 12$ & $-178.6(7)$ \\
\hline $\mathrm{C} 13-\mathrm{N} 1-\mathrm{C} 12-\mathrm{C} 7$ & $171.7(6)$ & $\mathrm{C} 6-\mathrm{C} 7-\mathrm{C} 8-\mathrm{C} 9$ & $-177.5(6)$ \\
\hline $\mathrm{C} 13-\mathrm{N} 1-\mathrm{C} 12-\mathrm{C} 11$ & $-6.5(11)$ & $\mathrm{C} 6-\mathrm{C} 7-\mathrm{C} 12-\mathrm{N} 1$ & $-0.1(7)$ \\
\hline $\mathrm{N} 1-\mathrm{C} 1-\mathrm{C} 2-\mathrm{C} 3$ & $179.7(6)$ & $\mathrm{C} 6-\mathrm{C} 7-\mathrm{C} 12-\mathrm{C} 11$ & $178.3(5)$ \\
\hline $\mathrm{N} 1-\mathrm{C} 1-\mathrm{C} 6-\mathrm{C} 5$ & $179.9(5)$ & $\mathrm{C} 8-\mathrm{C} 7-\mathrm{C} 12-\mathrm{N} 1$ & $-179.6(6)$ \\
\hline $\mathrm{N} 1-\mathrm{C} 1-\mathrm{C} 6-\mathrm{C} 7$ & $2.0(7)$ & $\mathrm{C} 8-\mathrm{C} 7-\mathrm{C} 12-\mathrm{C} 11$ & $-1.2(9)$ \\
\hline $\mathrm{C} 2-\mathrm{C} 1-\mathrm{C} 6-\mathrm{C} 5$ & $-1.2(10)$ & $\mathrm{C} 12-\mathrm{C} 7-\mathrm{C} 8-\mathrm{C} 9$ & $1.7(9)$ \\
\hline $\mathrm{C} 2-\mathrm{C} 1-\mathrm{C} 6-\mathrm{C} 7$ & $-179.1(6)$ & $\mathrm{C} 7-\mathrm{C} 8-\mathrm{C} 9-\mathrm{C} 10$ & $-1.3(10)$ \\
\hline $\mathrm{C} 6-\mathrm{C} 1-\mathrm{C} 2-\mathrm{C} 3$ & $1.1(10)$ & $\mathrm{C} 8-\mathrm{C} 9-\mathrm{C} 10-\mathrm{C} 11$ & $0.1(12)$ \\
\hline $\mathrm{C} 1-\mathrm{C} 2-\mathrm{C} 3-\mathrm{C} 4$ & $-0.8(11)$ & $\mathrm{C} 9-\mathrm{C} 10-\mathrm{C} 11-\mathrm{C} 12$ & $0.4(12)$ \\
\hline $\mathrm{C} 2-\mathrm{C} 3-\mathrm{C} 4-\mathrm{Br} 1$ & $-179.2(6)$ & $\mathrm{C} 10-\mathrm{C} 11-\mathrm{C} 12-\mathrm{N} 1$ & $178.1(7)$ \\
\hline $\mathrm{C} 2-\mathrm{C} 3-\mathrm{C} 4-\mathrm{C} 5$ & $0.6(11)$ & $\mathrm{C} 10-\mathrm{C} 11-\mathrm{C} 12-\mathrm{C} 7$ & $0.1(10)$ \\
\hline
\end{tabular}

Symmetry codes: (i) $-x+1,-y+1,-z+1$; (ii) $-x+1, y+1 / 2,-z+1 / 2$; (iii) $x-1 / 2, y,-z+1 / 2$; (iv) $-x+1 / 2, y+1 / 2, z$; (v) $x-1 / 2,-y+1 / 2,-z+1$; (vi) $-x+1, y-1 / 2$, $-z+1 / 2$; (vii) $-x+1,-y,-z+1$; (viii) $x, y+1, z$; (ix) $-x+3 / 2, y+1 / 2, z$; (x) $x, y-1, z$; (xi) $-x+3 / 2, y-1 / 2, z$; (xii) $x+1 / 2, y,-z+1 / 2$; (xiii) $-x+1 / 2, y-1 / 2, z$; (xiv) $x+1 / 2,-y+1 / 2,-z+1$. 
Hydrogen-bond geometry $\left(A,{ }^{\circ}\right)$

$\mathrm{Cg} 1$ are the centroids of the $\mathrm{N} 1 / \mathrm{C} 1 / \mathrm{C} 6 / \mathrm{C} 7 / \mathrm{C} 12$ and $\mathrm{C} 1-\mathrm{C} 6$ rings, respectively.

\begin{tabular}{lllll}
\hline$D-\mathrm{H} \cdots A$ & $D-\mathrm{H}$ & $\mathrm{H} \cdots A$ & $D \cdots A$ & $D-\mathrm{H} \cdots A$ \\
\hline $\mathrm{C} 8-\mathrm{H} 8 \cdots C g 1^{\mathrm{ii}}$ & 0.93 & 2.81 & $3.637(7)$ & 149 \\
$\mathrm{C} 11-\mathrm{H} 11 \cdots C g 2^{\mathrm{ix}}$ & 0.93 & 3.01 & $3.922(8)$ & 167 \\
\hline
\end{tabular}

Symmetry codes: (ii) $-x+1, y+1 / 2,-z+1 / 2$; (ix) $-x+3 / 2, y+1 / 2, z$. 